\title{
Superwind Galaxies at High Redshift: the Case of LAE J1044-0130
}

\author{
Masaru Ajiki, Shinobu S. Fujita, Yasuhiro Shioya, Tohru Nagao, \\ Takashi Murayama, Yoshiaki Taniguchi \\ Astronomical Institute, Graduate School of Science, Tohoku University, \\ Aramaki, Aoba, Sendai 980-8578, Japan
}

\begin{abstract}
Superwind galaxies at high-redshift are now considered to be the important clue for understanding the interaction and evolution of both galaxies and intergalactic matter. In order to improve our knowledge of superwind galaxies at high redshift, a large sample of superwind candidates is needed. During the course of our new search for Lyman alpha emitters at $z=5.7$, we have found a candidate superwind galaxy, LAE J1044-0130, at $z=5.69$. The emission-line profile of this object is asymmetric, showing excess red-wing emission and the observed broad line width $(=340 \mathrm{~km} / \mathrm{s})$. In this poster, we report detailed observed properties of LAE J1044-0130 as an example of a superwind galaxy.
\end{abstract}

\section{Introduction}

Galactic superwinds are now considered to be one of the key issues for understanding the interaction and evolution of both galaxies and intergalactic matter (e.g. Taniguchi \& Shioya 2000). In order to improve our knowledge of galactic superwinds at high redshift, a large sample of superwind candidates at $z>3$ is needed. During the course of our new search for Ly $\alpha$ emitters at $z \approx 5.7$, we have found a candidate superwind galaxy, LAE1044-0130 at $z=5.69$. In the following section, we report on its observed properties.

\section{Properties of LAE1044-0130}

The optical thumb-nail images of LAE J1044-0130 obtained with Suprime-Cam on the $8.2 \mathrm{~m}$ Subaru Telescope are given in the left panel of Fig. 1. As shown in this figure, LAE J1044-0130 is clearly seen only in the NB816 image; the observed equivalent width is $E W_{\text {obs }}>310 \AA$. The NB816 image reveals that LAE J1044-0130 is spatially extended.

The spectrum of LAE J1044-0130 obtained with ESI on Keck II are shown the right panel of Fig. 1. The emission-line profile at $8130 \AA$ is confirmed to slightly asymmetric, showing a cutoff at wavelengths shortward of the line peak. The Ly $\alpha$ emission line at $8129.3 \pm 3.0 \AA$ yields a redshift of $5.687 \pm 0.002$. The rest-frame equivalent width of the putative $\operatorname{Ly} \alpha$ emission is estimated to be $E W_{0}>46 \AA$. The observed Ly $\alpha$ flux is $(1.49 \pm 0.33) \times 10^{-17} \mathrm{ergs} \mathrm{cm}^{-2} \mathrm{~s}^{-1}$. The result of two-component profile fitting is also shown in the right panel of Fig 

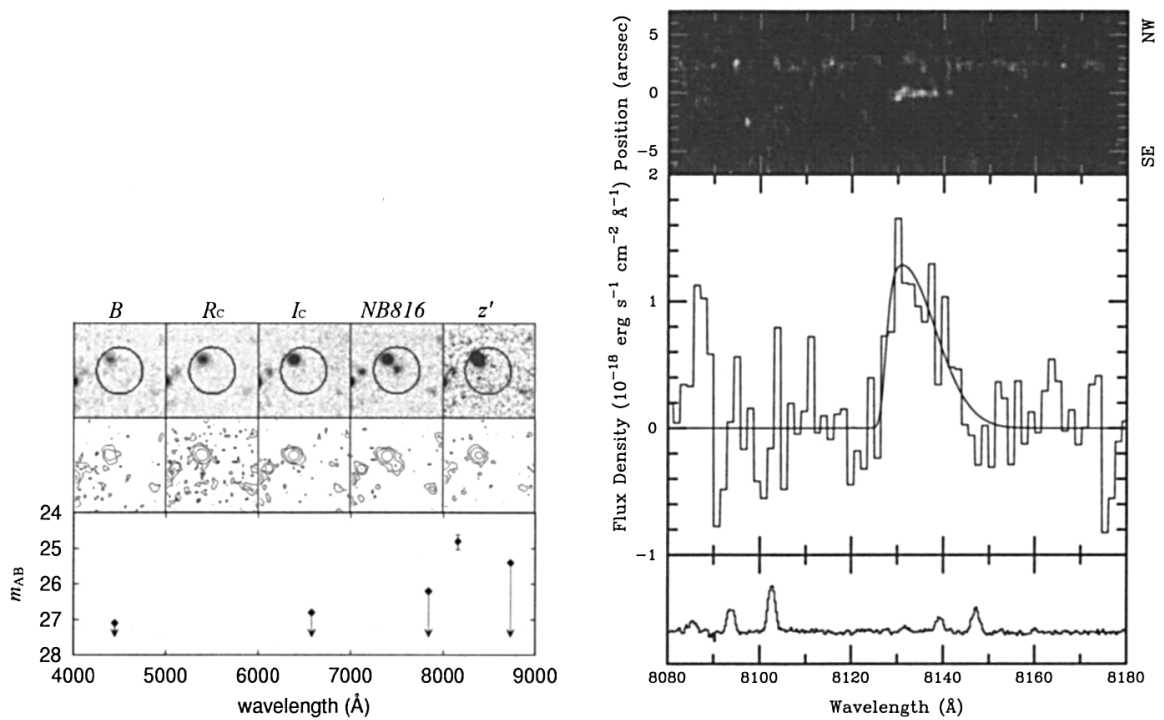

Figure 1. Left: Thumbnail images of LAE J1044-0130 (upper panel) also displayed as contours (middle panel). The angular size of the circle in each panel corresponds to $8^{\prime \prime}$. The lower panel shows the spectral energy distribution (in magnitudes). Right: The optical spectrogram (upper panel) and one-dimensional spectrum (middle panel) of LAE J1044-0130 obtained with ESI on Keck II $(R \sim 3400)$; note that fivepixel binning was applied to the one-dimensional spectrum. The model profile fit is shown by the thick solid curve (see text). Sky (OH airglow) emission lines are shown in the lower panel.

1 (thick curve). The FWHM of the Ly $\alpha$ emission is measured to be $340 \pm 110$ $\mathrm{km} \mathrm{s}^{-1}$ and the full width at zero intensity (FWZI) is estimated to be $890 \pm 110$ $\mathrm{km} \mathrm{s}^{-1}$. These properties are similar to those of the Ly $\alpha$ emitter at $z=5.190$, J123649.2+621539, found by Dawson et al. (2002).

Acknowledgments. We would like to thank both the Subaru and Keck Telescope staff for their invaluable help. This work was financially supported in part by the Ministry of Education, Culture, Sports, Science, and Technology (Nos. 10044052, and 10304013).

\section{References}

Ajiki, M. et al. 2002. ApJ, 576, L25

Dawson, S., Spinrad, H., Stern, D., Dey, A., van Breugel, W., De Vries, W., \& Reuland, M. 2002, ApJ, 570, 92

Taniguchi, Y., \& Shioya, Y. 2000, ApJ, 547, 146 